\title{
Recombinant production and purification of the human protein Tau
}

\author{
Luca Ferrari ${ }^{1,2}$, Stefan G.D. Rüdiger ${ }^{* 1,2}$ \\ ${ }^{1}$ Cellular Protein Chemistry, Bijvoet Center for Biomolecular Research, Utrecht University, \\ Padualaan 8, 3584 CH Utrecht, The Netherlands \\ ${ }^{2}$ Science for Life, Utrecht University, Padualaan 8, 3584 CH Utrecht, The Netherlands \\ * Corresponding author
}

Keywords: Neurodegeneration, Alzheimer's Disease, Protein Purification, Intrinsically Disordered Protein, Protein Aggregation

\begin{abstract}
Tau protein is a microtubule-stabilizing protein whose aggregation is linked to Alzheimer's Disease and other forms of dementia. Tau biology is at the heart of cytoskeletal dynamics and neurodegenerative mechanisms, making it a crucial protein to study. Tau purification, however, is challenging as Tau is disordered, which makes it difficult to produce in recombinant system and is degradationprone. It is thus challenging to obtain pure and stable preparations of Tau. Here, we present a fast and robust protocol to purify Tau recombinantly in Escherichia coli. Our protocol allows purifyig Tau either tag-less free or FLAGtagged at its $\mathrm{N}$-terminus. By exploiting a cleavable affinity tag and two anion exchange columns, we obtained Tau is of high purity, stable and suitable for in vitro studies, including aggregation experiments that resemble neurodegenerative processes.
\end{abstract}




\section{INTRODUCTION}

Tau aggregation is the prominent signature of Alzheimer and other diseases termed tauopathies (1-3) It is linked to neuronal death and sufficient to induce neurodegeneration $(2,4)$. In Alzheimer, aggregation of Tau into fibrils correlates with cognitive impairment in humans Understanding Tau biology is thus of paramount importance to understand neurodegeneration.

Tau protein is predominantly expressed in the central nervous system, with six documented isoforms obtained from alternative splicing and developmentally regulated (4). Tau belongs to the family of microtubule associated proteins, or MAPs, collectively orchestrating microtubule assembly (6), This family of proteins act redundantly, as Tau null mice show no phenotype and have increased levels of the paralog MAP1A (7). Studies attempting to propose a role of Tau in axon growth yielded varying results, based on the cell lines investigated $(7,8)$. Recent data demonstrated how Tau and other MAPs compete to distribute cargos correctly ( (9)). Thus, Tau is bound to microtubules in physiological state, and its detachment from them is a necessary step preceding aggregation and pathology.

Tau binding to microtubules is highly dynamic and regulated by phosphorylation, with Tau having

more than 40 potential phosphorylation sites (10). Phosphorylation and consequent detachment from microtubule are thought to be the initial steps triggering Tau aggregation in dementia, however recent structural data show that phospho-sites do not have a structural role in the assembly of Tau aggregates (11, 12). Also, hyperphosphorylation is fundamental for hibernation of rodents but does not lead to dementia (6). Finally, some phosphorylation sites may have a protective role against Tau aggregation (13). Thus, Tau hyperphosphorylation cannot explain alone aggregation and disease progression.

Tau aggregation may be caused by the collapse of the chaperone network instead (14). Tau sequence itself endows the potential for Tau aggregation. Tau is a disordered protein of 441 residues in its longest isoform $(4,15)$. Tau $\mathrm{N}$ terminus composition enhance its solubility, with few hydrophobic residues (10.6 non hydrophobic residues per hydrophobic one (16)). On the contrary, microtubule binding region has higher hydrophobic content (4.4 non hydrophobic residues per hydrophobic one), and indeed is the region comprising the core of amyloid structures of Alzheimer's and Pick's Disease $(11,12)$. Although Tau is soluble and dynamic, its own sequence has the potential to convert to toxic folds. Surprisingly, it is hard to pinpoint the 
exact role of aggregates in neurodegeneration (17) Thus, Tau pathology is left with many unresolved issues, making it necessary to develop new approaches to study it. These approaches include generating stable and reliable preparations of Tau. Obtaining pure Tau preparations is however challenging, as Tau disordered nature makes it vulnerable to protease action (15). Tau stability and purity are essential to perform a variety of assays, for instance aggregation experiments recapitulating neurodegeneration. Current available protocols focus on purifying tag-less versions, however this may limit downstream applications relying on Tau recognition by an antibody, such as pull-downs or western blot detection $(18,19)$.

Here, we present an alternative protocol to obtain recombinant Tau from Escherichia coli. Compared to previous protocol, we introduced a cleavable tag $\mathrm{His}_{6}-\mathrm{Smt}$ at the $\mathrm{N}$ terminus to maintain a tag-less version of the protein. We introduced a second anion exchange purification step to remove the tag and to further decrease impurities. Despite this extra purification step, the resulting protocol was still fast, meaning that Tau purification - from cell lysate to pure protein - takes 1 to 2 days, based on the purified variant. We show that the protocol robustly purified also a FLAG-tagged version of Tau at the N-terminus. This variant can be used for instance to perform pull-down experiments or to favour Tau detection with an antibody. We obtained Tau preparations of high purity and yields (between 1 and $3 \mathrm{mg}$ of proteins per $1 \mathrm{I}$ of bacterial culture), suitable for aggregation experiments and other applications.

\section{RESULTS}

\section{Design of biologically relevant Tau variants}

The Tau protein consists of several functionally relevant regions (Fig. 1A). The N-terminal region (A2L243) is important for Tau sorting in the cell and is endowed with a proline-rich domain, whose isomerization is possibly linked to increased aggregation propensity (20). After this stretch, the repeat domains follow (Q244-N368), which consists of fourpseudo-repeats. They are responsible for binding to microtubules and participating to the formation of amyloid structures, in particular the $3^{\text {rd }}$ and $4^{\text {th }}$ repeats $(11,12)$. The C-terminal region (K369-L441) is physiologically removed when Tau is secreted in the cerebrospinal fluid (21). We aimed to produce three types of Tau constructs: wild type, with pseudo+phosphorylated sites or with increased aggregation propensity.

A major drawback of studying Tau dynamics is that phosphorylation studies are difficult to perform, as kinases phosphorylase 
stochastically a subgroup of phosphorylation sites, resulting in heterogenous populations of phospho-Tau species. To this end, we designed a pseudophosphorylated Tau construct inspired by previous studies (Tau ${ }^{\mathrm{pp}}$, Fig. 1A), where replacement of serines with glutamates at defined positions 262, 293, 324, 356 mimics the phosphorylation sites of Tau relevant for disease (22). This approach removes the need for kinases and still offers a functionally relevant protein for studying the impact of negative charges at defined positions.
A second hurdle relates to Tau aggregation dynamics. Indeed, aggregation of full-length Tau takes from several days to up to a week, a time scale where many biological samples become unstable (19). To this end, we generated a truncation of Tau comprising the four repeats (Tau-Q244-E372; Tau Repeat Domain, Tau-RD, also referred to as $\mathrm{K} 18$ in the literature ( (23)), with reported faster aggregation rates, in the time scale of hours (24). This truncation is an established disease model, causing neurodegeneration in mice (25).
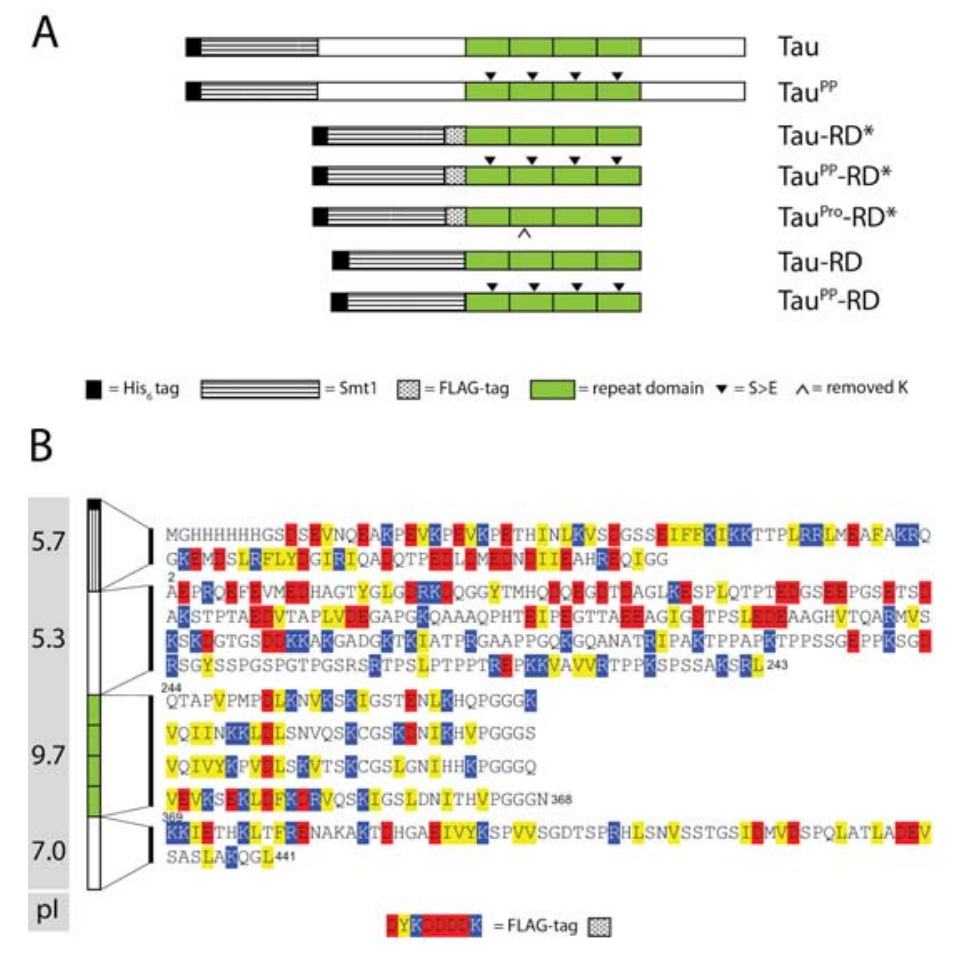

Figure 1. Tau variants used in this study.

a. Scheme of Tau wild type and Tau variants used in this study.

PP: replacement of serines with glutamates at positions 262, 293, 324, 356 (S>E).

RD: Repeat Domain.

*: FLAG-tag.

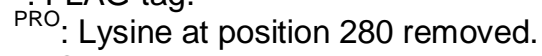

b. Overview of Tau sequence, hidrophobicity and charge distribution. $\mathrm{His}_{6}$-Smt1-Tau sequence colored by hydrophobicity (yellow), negative charges (red) and positive charges (blue). Theoretical Isolectric points (pl) of each domain are shown in the grey box on the left. 
All of our constructs were designed with a cleavable $\mathrm{His}_{6}$-Smt tag at the $\mathrm{N}$-terminus. A hexahistidine tag serves as a purification tag, while Smt is a globular domain that favours protein production. After cleavage, Tau protein comprises all residues between $A 2$ and $L 441$ (Fig. 1B). Regarding Tau-RD, we added a FLAG tag (DYKDDDDK) at the $\mathrm{N}$ terminus, immediately C-terminal of the cleavage site of Smt1, composed of two Glycines (GG). Thus, the tag is retained after cleavage. It serves for downstream applications, such as western blot detection or pull-down experiments, and positioning at the $\mathrm{N}$-terminus avoids interference with the aggregating regions present toward the C-terminus. Taken together, our designed constructs are suitable to study Tau phosphorylation, aggregation and other aspects of Tau biology.

\section{Tau sequence properties define strategies for purification}

When analysing the amino acidic distribution in Tau, we noted asymmetry in the distribution of charges (Fig. 1B). We calculated the isoelectric point ( $\mathrm{pl}$ ) of various segments of Tau based on their composition. While the $\mathrm{N}$ - and $\mathrm{C}$ terminal regions (A2-L243 and K369-L441) have a pl of 5.7 and 7.0 respectively, the repeat domain has a pl of 9.7. This implies that at the physiological $\mathrm{pH}$ of the cell the $\mathrm{N}$ terminal region is negatively charged, while the repeat domain is positively charged, and the Cterminal region has a near-neutral net charge. Of note, the $\mathrm{His}_{6}-\mathrm{Smt}$ tag has a pl of 5.3, being thus negatively charged at physiological $\mathrm{pH}$. These differentially charged domains can be exploited for binding to different ion exchanges columns, to achieve greater purity of the sample.

\section{Production and Purification of full-length Tau}

First, we aimed to develop a strategy to purify full-length Tau (Fig. 2A). First, E. coli BL21 DE3 Rosetta 2 cells were transformed with a plasmid encoding for a Tau variant. We chose this cellular strain as it encodes a T7 polymerase necessary to transcribe Tau plasmid. It also encodes for suppressor tRNAs for rare codons, which appear frequently in the human Tau construct. Cells were then induced with Isopropyl $\beta$-D-1thiogalactopyranoside (IPTG) at an optical density (OD) at $600 \mathrm{~nm}$ of 0.8 , and they were cultured over night at $18^{\circ} \mathrm{C}$. Next, they were lysed. Subsequently we heated the lysate at $95^{\circ} \mathrm{C}$. This is a key step to increase sample stability as Tau is an intrinsically disordered protein (IDP). Tau cannot, therefore, lose potentially folded structure. The majority of proteins, including proteases, became insoluble and precipitated under these conditions, whereas Tau remained in the supernatant due to its high solubility and lack of folded domains, which could become potentially 
aggregation-prone after unfolding. Tau-containing supernatant was loaded on an affinity purification column, to which it bound thanks to the $\mathrm{His}_{6}$ tag at the $\mathrm{N}$-terminus of the protein. After elution by an imidazole gradient, the protein was further purified with an anion exchange column. Tau was then eluted in a salt gradient. We pooled the Tau

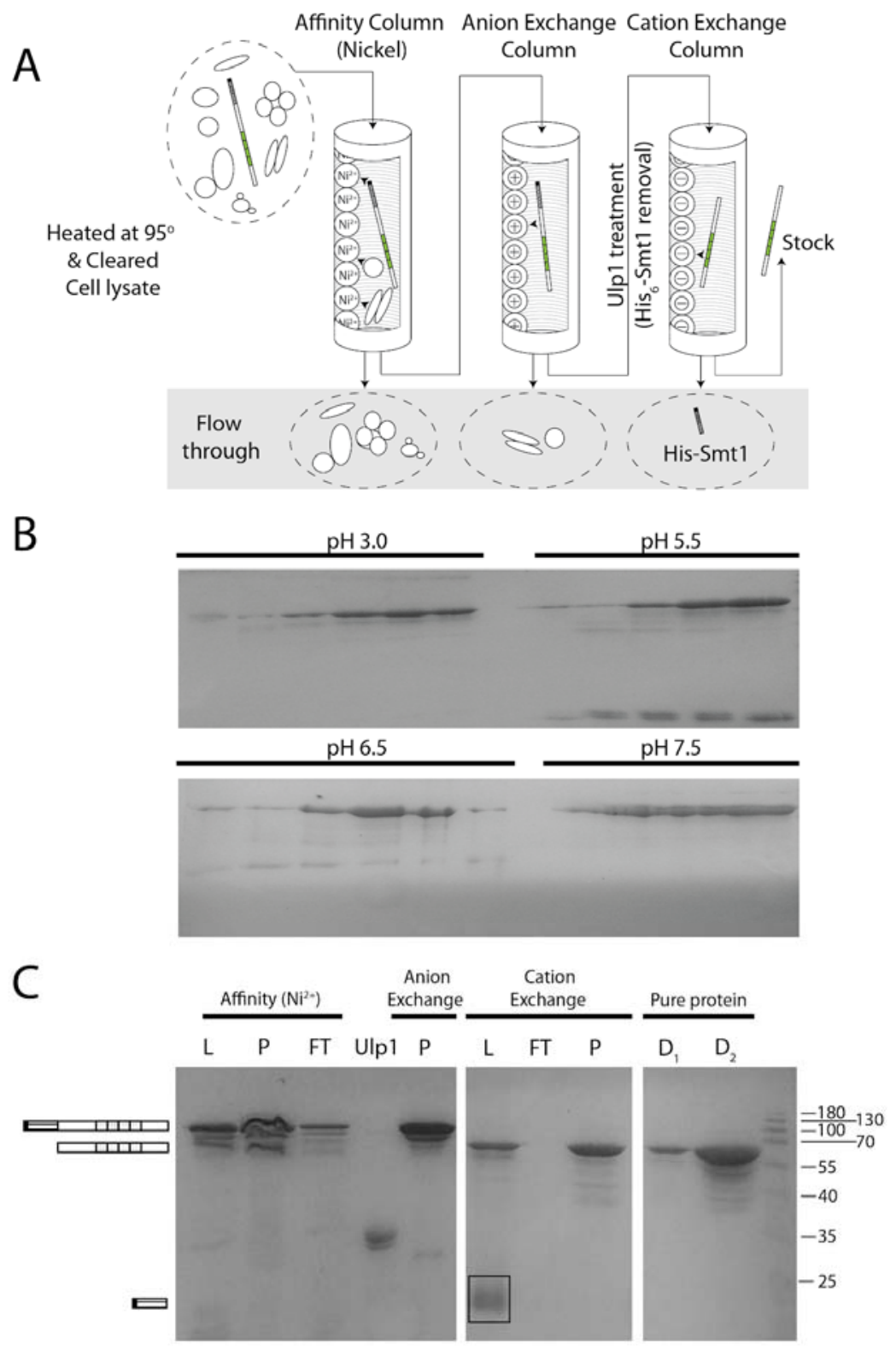

Figure 2. Purification of full-length Tau

a. Purification scheme of full-length Tau.

b. pH scouting to maximize Tau purity. 15\% SDS-PAGE gel showing results of pH scouting with cation exchange columns. Four pHs were tested, fractions representative of the whole elution peak were collected and loaded on gel.

c. Purification of wild type Tau. 15\% SDS-PAGE gel showing increased purity of Tau protein as purification proceeds. L: samples loaded on column; P: elution peak; FT: flow through; D1: dilution 1; D2: dilution 2. Boxed band indicates removed SUMO-tag, numbers on the right side of the gel indicate molecular weights in KDa. On the left: schemes of protein segments from Fig. $1 \mathrm{~A}$ corresponding to different gel bands. 
containing fractions and cleaved off the $\mathrm{His}_{6}-\mathrm{Smt}$ by adding Ulp1 hydrolase to obtain an untagged Tau species. The resulting protein mix was then loaded on a cation exchange column, where effective separation of Tau from the tag took place upon elution with a salt gradient, due to the different net charges of Tau (positively charged

A

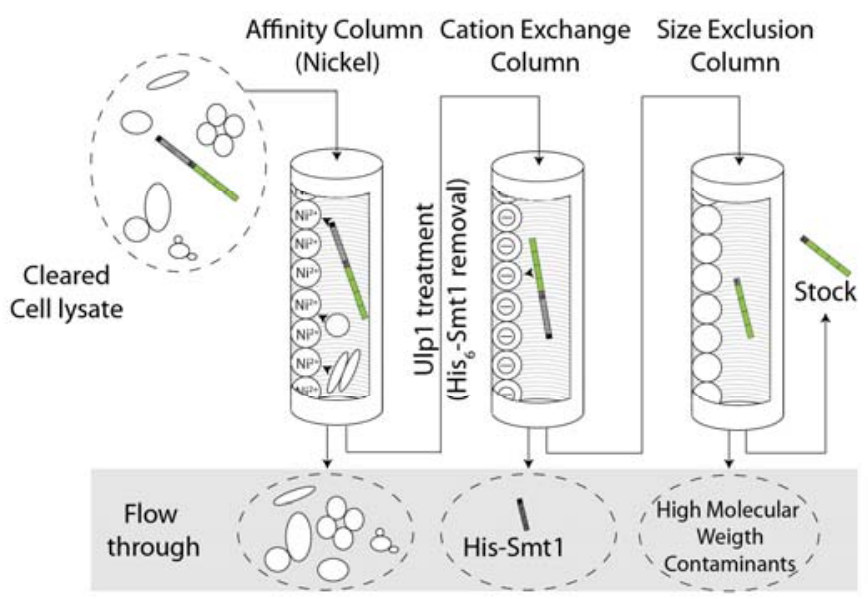

B
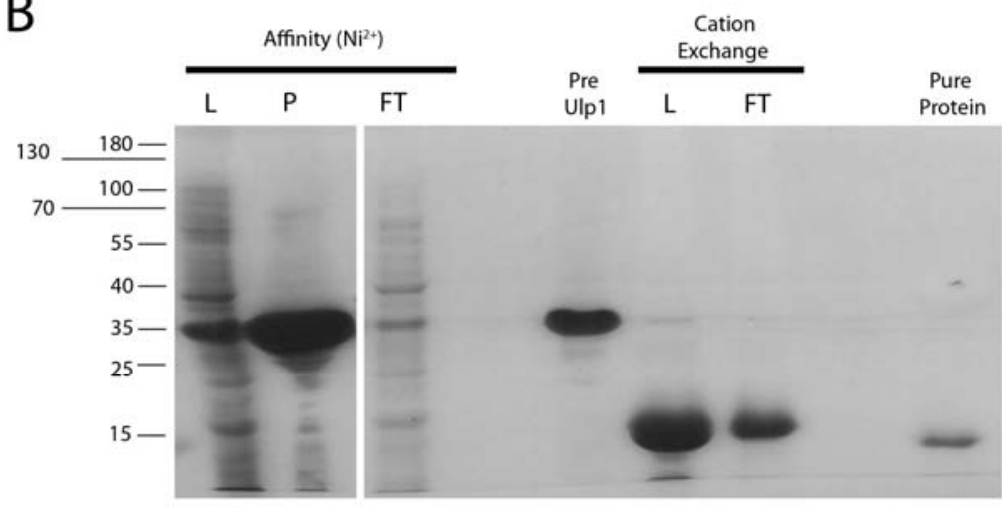

C

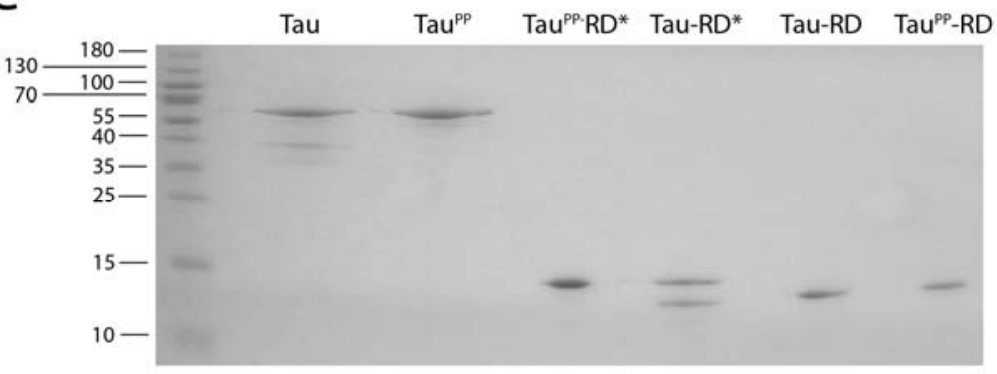

Figure 3. Purification of Tau Repeat Domain.

a. Purification scheme of Tau-RD.

b. Purification of wild type Tau-RD*. $12 \%$ SDS-PAGE gel showing increased purity of Tau Pro$\mathrm{RD}^{*}$ protein as purification proceeds. L: samples loaded on column; P: elution peak; FT: flow through. Numbers on the left side of the gel indicate molecular weights in KDa.

c. Robust purification of Tau and Tau-RD variants. 15\% SDS-PAGE gel showing end products of purifications of different Tau variants. Numbers on the left side of the gel indicate molecular weights in $\mathrm{KDa}$. 
Tau-RD allowed binding to the column) and $\mathrm{His}_{6}$-Smt (negatively charged, repelled by the column and found in flow through). This was a critical step to achieve high purity, as differences in the equilibration $\mathrm{pH}$ of the cation column yielded less pure eluates, possibly favouring the binding of differentially charged Tau truncation and/or impurities (Fig. 2B). While an eluting $\mathrm{pH}$ of 7.5 resulted in pure protein, $\mathrm{pH}$ of 3.0 , 5.5 and 6.5 yielded less pure protein. We monitored the purification scheme and sample quality by SDS-PAGE (Fig. 2C), highlighting the high degree of purity of the final product. We obtained between 5 and $10 \mathrm{mg}$ of proteins per 3.2 I of bacterial culture $(n=3)$.

\section{Robust purification of Tau variants}

Next we set out to adapt our protocol for purifying Tau-RD. Remarkably, we could not retrieve protein after heating. We concluded that the highly charged terminal regions increased overall solubility, in line with the observation that Tau$\mathrm{RD}$ is more prone to form fibrils than the full length protein. We consequently adapted the protocol by removing the boiling step (Fig. 3A). As Tau-RD has a net positive charge at physiological $\mathrm{pH}$, we also avoided the anion exchange purification step. To compensate for lacking these two steps, we added a size exclusion chromatography step downstream of the purification scheme to remove eventual high molecular weight contaminants. Our adapted protocol resulted in highpurity Tau-RD, as assessed by SDS-PAGE (Fig. 3B). We obtained between 4.0 and $8.7 \mathrm{mg}$ of proteins per 3.2 I of bacterial culture $(n=5)$.

Importantly, the two protocols presented for full-length Tau and Tau-RD were extended to other Tau variants, containing for instancepseudo-phosphorylated residues or FLAG-tag, nonetheless yielding pure proteins (Fig. $\mathbf{3 C}$ ). In the case of FLAG-tagged constructs, $\mathrm{His}_{6}-\mathrm{Smt}$ cleavage was extended overnight, as a one-hour treatment was not sufficient to cleave the protein completely. Thus, we could further adapt our protocol developed for full-length Tau to other Tau variants.

\section{Purified Tau aggregates successfully}

We tested the quality of our purified proteins by employing them in an established aggregation assay (26). The negatively charged polymer initiates Tau fibrillation, which is a reliable and reproducible method to study kinetics of Tau aggregation and other aspects linked to Tauinduced neurodegeneration. First, we monitored via SDS-PAGE the aggregation propensity over time of full-length $\mathrm{Tau}$ in the presence of increased amounts of heparin (Fig. 4A). We observed the appearance of SDS-insoluble aggregates after 6 days of aggregation, at the optimal stoichiometry of four molecules of Tau per molecule of heparin. This is an established stoichiometry to 
obtain Tau aggregates resembling Alzheimer's Paired Helical Filaments (27). We did not observe formation of aggregates at sub-stoichiometric Tau:heparin ratios during the time frame of our experiments, whereas excess of heparin interfered with Tau running on the electrophoresis gel, probably due to the high number of negative charges decorating heparin. Thus, our experiment confirmed that our Tau preparation have similar aggregation properties as Tau batches purified with other protocols $(18,19)$. Compared to other protocols, our purification setup allows purifying Tau variants with different biochemical properties, such as increased aggregation propensity, pseudo-phosphorylated sites or a FLAG-tag at their Nterminus. This versatility does not come at the expense of yields,
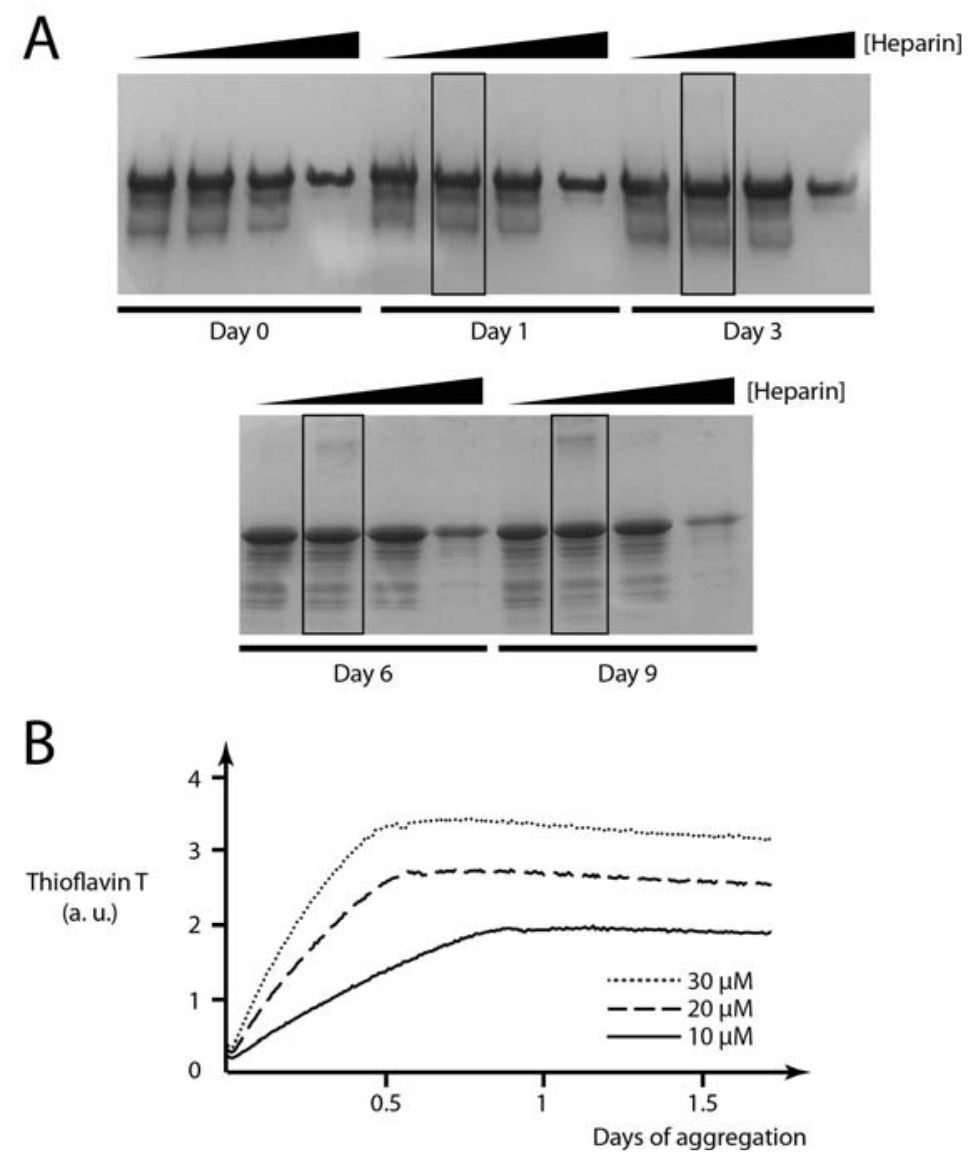

Figure 4. Heparin induces aggregation of Tau variants.

a. Optimal stoichiometry for heparin-induced Tau aggregation is 1:4 (heparin-Tau). 12\% SDS-PAGE gel showing appearance of Tau SDS-resistant protein aggregates over time upon addiction of heparin. Gradients indicate increasing heparin concentrations, boxed lanes indicate optimal heparin:Tau stoichiometry 1:4.

b. Higher concentration accelerates aggregation of $\mathrm{Tau}^{\mathrm{Pro}}-\mathrm{RD}^{*}$. Thioflavin $\mathrm{T}$ assay showing aggregation of $\mathrm{Tau}^{\mathrm{PrO}}-\mathrm{RD}^{*}$ at 3 different concentrations (heparin:Tau stoichiometry kept constant at 1:4). Fluorescence correlates with amyloid content and is expressed in arbitrary unit (a. u.). 
purity and purification time. Tau preparations were stable even after a week of incubation at $37^{\circ} \mathrm{C}$, proving that our purification procedure is highly effective in removing contaminant proteases (Fig. 4A).

We further tested the robustness of our protocol by inducing aggregation of Tau-RD domain. We used the Thioflavin $T$ assay to monitor kinetics of aggregation over time. This is an established technique to study aggregation dynamics, in which a fluorescent dye binds to fibrils but not monomers of Tau, resulting in a fluorescent signal allowing monitoring aggregation over time (28). We kept the Tau:heparin ratio as $4: 1$, this time changing the concentration of monomeric Tau-RD at the beginning of the aggregation, to test (Fig. 4B). Increased concentrations of Tau accelerated Tau aggregation, consistent with the proposed model of nucleation-elongation proposed for heparin-induced Tau aggregation (29). Thus, our protocol yields Tau variants whose aggregation dynamics are in agreement with published material.

\section{DISCUSSION}

Here, we presented a protocol to purify Tau species after recombinant production in Escherichia coli. Purification of intrinsically disordered proteins is challenging and may require tedious optimization (30). Our protocol allows Tau purification with standard purification equipment and buffers. Compared to previous Tau purification methods, our protocol allows to purify Tau either tag-less or with a tag of choice, thanks to the removable $\mathrm{His}_{6}$-Smt tag at the $\mathrm{N}$ terminus of the protein $(18,19)$. Tau distribution of charges allows effective purification and removal of the $\mathrm{His}_{6}$-Smt. Indeed, Tau can both bind to an anion and cation exchange column, the latter used to effectively separate the tag from the purified protein. Most of bacterial proteome has a distribution of isoelectric points lower than 7, thus is negatively charged at physiological $\mathrm{pH}$ and removed either by the Nickel column or by the cation exchange column (31). A sub-fraction of the proteome, mostly DNA and RNA binding proteins, is positively charged and thus effectively removed by the anion exchange column. Overall, a double ion-exchange column approach allows effective purification of Tau protein.

Interestingly, the elution buffer of the cation exchange column greatly influences the purity of Tau fulllength (Fig. 2B). This is probably due to the asymmetric distribution of charges throughout the random coils of Tau protein, exposing different charged patches that bind to the column as $\mathrm{pH}$ changes. This distribution is also important for physiological functions of Tau, as the high pl of Tau-RD is necessary for binding to microtubules. In contrast, the $\mathrm{N}$ terminal regions of Tau are negatively charged, similar 
to most cytoplasmic proteins, such that they remain dynamic and exposed even when bound to microtubules (4).

We could not apply the same exact protocol to Tau-RD truncations, as they lack the $\mathrm{N}$-terminus region necessary to bind to the anion exchange column. Tau-RD could not be retrieved after the boiling step, suggesting that the $\mathrm{N}$ - and $\mathrm{C}$ termal regions drastically increase Tau solubility. Of note, a study presenting a general purification protocol for disordered proteins based on boiling cell lysates suggests that Tau-RD behaves atypically compared to other disordered segments (32). These differences in solubility may also have had an influence on the evolution of physiologically relevant Tau truncations. Indeed, Tau truncations at the $\mathrm{N}$-terminus are found in humans in physiological conditions, whereas C-terminal truncations were not detected (21). It is tempting to speculate that their higher propensity to aggregate negatively selected them, in favour of more soluble N-terminal truncations.

Our designed Tau constructs, both full-length and $\mathrm{RD}$, aggregate with an optimal Tau:heparin stoichiometry of $4: 1$, in agreement with published protocols, suggesting that our Tau constructs can be used to model aggregation in Alzheimer and other forms of dementia (28, 29). Indeed, we used N-terminally FLAG-tagged Tau-RD to create
Alzheimer-like fibrils, which we could later disaggregate with the human Hsc70 chaperone system (33). To conclude, we present a protocol yielding pure and stable Tau constructs suitable for a wide array of in vitro studies.

\section{ACKNOWLEDGMENTS}

We are grateful to Ineke Braakman for continuous support. We thank Madelon Maurice for collaboration in the Initial Training Network "WntsApp" (No. 608180)], supported by Marie-Curie Actions of the 7th Framework programme of the EU. SGDR was further supported by the Internationale Stichting Alzheimer Onderzoek (ISAO; project "Chaperoning Tau Aggregation"; No. 14542) and a ZonMW TOP grant ("Chaperoning Axonal Transport in neurodegenerative disease"; No. 91215084).

\section{AUTHOR CONTRIBUTIONS}

S.G.D.R. and L.F. conceived the study; S.G.D.R., L.F. and F.G.F. planned experiments; L.F., W.J.C.G., M.v.W., R.K., K.K. and L.v.B. did experiments; L.F., W.J.C.G., M.v.W., R.K., K.K. and L.v.B. analysed data; S.G.D.R. and L.F. wrote the manuscript, with contributions of all authors.

\section{COMPETING INTERESTS}

The authors declare no competing interests.

\section{MATERIALS AND METHODS Isoelectric points calculation}

Theoretical isoelectric points were calculated using ProtParam tool (https://web.expasy.org/protparam/). 


\section{Purification of Tau variants}

We designed two types of Tau constructs, full-length and Repeat Domain (Fig. 1A). We propose two purification protocols, based on the variants to be purified.

\section{Purification of human full-length Tau}

Following, a step-by step procedure to purify human full-length Tau:

1. Human Tau and Tau ${ }^{\mathrm{PP}}$ were overproduced recombinantly in E. coli BL21 Rosetta 2 (Novagen). Cells were induced with $0,15 \mathrm{mM}$ Isopropyl $\quad \beta$-D-1thiogalactopyranoside (IPTG, Sigma) at Optical Density at $600 \mathrm{~nm}$ (OD600) of 0.8 and cultured over night at $18^{\circ} \mathrm{C}$.

2. Cells were harvested, flashfrozen in liquid nitrogen and stored at $-80^{\circ} \mathrm{C}$ until further usage.

3. Pellets were thawed in a water bath at $37^{\circ} \mathrm{C}$ and resuspended in $50 \mathrm{mM}$ HEPES-KOH pH 8.5 (SigmaAldrich), $50 \mathrm{mM} \mathrm{KCl}$ (SigmaAldrich), $1 / 2$ tablet $/ 50 \mathrm{ml}$ EDTA-free protease inhibitor (Roche), $5 \mathrm{mM} \quad \beta$ mercaptoethanol (SigmaAldrich).

4. Cells were disrupted by an EmulsiFlex-C5 cell disruptor (Avestin).

5. Lysate was cleared by centrifugation, filtered with a
$0.22 \mu \mathrm{m}$ polypropylene filter (VWR) and supernatant was purified using an ÄKTA Purifier chromatography system (GE Healthcare).

6. Lysate was loaded onto a POROS 20MC (Thermo Fischer Scientific) affinity purification column in $50 \mathrm{mM}$ HEPES- $\mathrm{KOH} \mathrm{pH} 8.5,50 \mathrm{mM}$ $\mathrm{KCl}, \quad 5 \quad \mathrm{mM} \quad \beta$ mercaptoethanol, eluted with a $0-100 \%$ linear gradient $(5$ Column Volumes, CV) of 0.5 $M$ imidazole.

7. Fractions of interest were collected, diluted to salt concentration $<100 \mathrm{mM}$ and loaded onto a POROS $20 \mathrm{HQ}$ (Thermo Fischer Scientific) anion exchange column equilibrated with $50 \mathrm{mM}$ HEPES-KOH pH 8.5. Protein was eluted with a $0-100 \%$ linear gradient (15 CV) of $1 \mathrm{M}$ $\mathrm{KCl}$ (Carl Roth).

8. The $\mathrm{His}_{6}$-Smt tag was removed by Ulp1 treatment (purified in house), shaking at $4^{\circ} \mathrm{C}$ for 30 minutes.

9. Protein was diluted to salt concentration $<100 \mathrm{mM}$ and loaded onto a POROS $20 \mathrm{HS}$ (Thermo Fischer Scientific) cation exchange column equilibrated with $50 \mathrm{mM}$ HEPES-KOH pH 7.5. Protein was eluted with a $0-100 \%$ linear gradient (15 CV) of $1 \mathrm{M}$ $\mathrm{KCl}$ (Carl Roth).

10. Fractions of interest were further concentrated to the desired final concentration 


using a concentrator
(Vivaspin, cut-off $5 \mathrm{kDa}$ ).
1. Protein concentration was
determined using an ND-
1000
spectrophotometer
(Nanodrop Technologies)
based on the theoretical
extinction coefficient at 280
nm and purity was assessed
by SDS-PAGE. Protein was
aliquoted and stored at -
$80^{\circ} \mathrm{C}$.

\section{Purification of human Tau-RD}

Following, a step-by step procedure to purify human Tau-RD:

1. Human Tau-RD*, Tau ${ }^{\mathrm{PP}}-\mathrm{RD}^{*}$, Taupro-RD*, Tau-RD, Tau ${ }^{\mathrm{PP}}$ $\mathrm{RD}$ were overproduced recombinantly in E. coli BL21 Rosetta 2 (Novagen). Cells were induced with $0,15 \mathrm{mM}$ IPTG (Sigma) at Optical Density of 0.8 and cultured over night at $18^{\circ} \mathrm{C}$.

2. Cells were harvested, flashfrozen in liquid nitrogen and stored at $-80^{\circ} \mathrm{C}$ until further usage.

3. Pellets were thawed in a water bath at $37^{\circ} \mathrm{C}$ and resuspended in $50 \mathrm{mM}$ HEPES-KOH pH 8.5 (SigmaAldrich), $50 \mathrm{mM} \mathrm{KCl} \mathrm{(Sigma-}$ Aldrich), $1 / 2$ tablet $/ 50 \mathrm{ml}$ EDTA-free protease inhibitor (Roche), $5 \quad \mathrm{mM} \quad \beta$ mercaptoethanol (SigmaAldrich).
4. Cells were disrupted by an EmulsiFlex-C5 cell disruptor (Avestin).

5. Lysate was cleared by centrifugation, filtered with a $0.22 \mu \mathrm{m}$ polypropylene filter (VWR) and supernatant was purified using an ÄKTA Purifier chromatography system (GE Healthcare).

6. Lysate was loaded onto a POROS 20MC (Thermo Fischer Scientific) affinity purification column in $50 \mathrm{mM}$ HEPES-KOH pH 8.5, 50 mM $\mathrm{KCl}, \quad 5 \quad \mathrm{mM} \quad \beta$ mercaptoethanol, eluted with a $0-100 \%$ linear gradient (5 Column Volumes, CV) of 0.5 $\mathrm{M}$ imidazole.

7. Fractions of interest were collected and concentrated in a buffer concentrator (Vivaspin, cut-off $10 \mathrm{kDa}$ ) to final volume of $3 \mathrm{ml}$.

8. The concentrated sample was desalted with a PD-10 desalting column (GH Healthcare) in $50 \mathrm{mM}$ HEPES-KOH pH 8.5, $1 / 2$ tablet $/ 50 \mathrm{ml}$ Complete protease inhibitor (Roche)) and $5 \mathrm{mM} \quad \beta$ mercaptoethanol.

9. The $\mathrm{His}_{6}$-Smt tag was removed by Ulp1 treatment, shaking at $4^{\circ} \mathrm{C}$ for 30 minutes (increased to 16 hours when purifying FLAG-containing Tau variants).

10. Next day, protein was diluted to salt concentration $<100$ $\mathrm{mM}$ and loaded onto a POROS 20HS (Thermo 
Fischer Scientific) cation exchange column equilibrated with $50 \mathrm{mM}$ HEPES-KOH pH 8.5. Protein was eluted with a $0-100 \%$ linear gradient (15 CV) of $1 \mathrm{M}$ $\mathrm{KCl}$ (Carl Roth).

11. Fractions of interest were collected and loaded onto a HiLoad 26/60 Superdex 200 pg (GE Healthcare Life Sciences) size exclusion column equilibrated with aggregation buffer (25 mM HEPES-KOH $\mathrm{pH}$ 7.5, Complete Protease Inhibitors (1/2 tablet/50 ml), $75 \mathrm{mM}$ $\mathrm{KCl}, 75 \mathrm{mM} \mathrm{NaCl}$ and $10 \mathrm{mM}$ DTT).

12. Fractions of interest were further concentrated to the desired final concentration using a concentrator (Vivaspin, cut-off $5 \mathrm{kDa}$ ).

13. Protein concentration was determined using an ND1000 UV/Vis spectrophotometer (Nanodrop Technologies) based on the theoretical extinction coefficient at 280 $\mathrm{nm}$ and purity was assessed by SDS-PAGE. Protein was aliquoted and stored at $80^{\circ} \mathrm{C}$.

\section{Sodium Dodecyl Sulfate - Polyacrylamide Electrophoresis (SDS-PAGE)}

$20 \mu \mathrm{L}$ of samples were collected for each purification/aggregation step of interest and were supplemented with $20 \mu \mathrm{L}$ of $2 \mathrm{x}$ sample buffer (0.625 M Tris pH 6.8 (SigmaAldrich), $12.5 \%$ glycerol (CARL ROTH), 1\% SDS (Bio-Rad), 0.005\% Bromophenol Blue (Bio-Rad), $5 \mathrm{mM}$ freshly added $\beta$-mercaptoethanol (Sigma-Aldrich). 15\% SDS gels were prepared (Separation buffer: $0.38 \mathrm{M}$ Tris pH 8.8, 15\% acrylamide(National Diagnostics), $0.1 \%$ SDS, $0.1 \%$ APS (SigmaAldrich), 0.04\% TEMED (SigmaAldrich), Stacking buffer: $0.125 \mathrm{M}$ Tris $\mathrm{pH} 6.8,4 \%$ acrylamide, $0.1 \%$ SDS, $0.075 \%$ APS, $0.1 \%$ TEMED) and run in $1 \mathrm{x}$ Laemmli buffer $(0.025$ $M$ Tris base $\mathrm{pH} 6.8,0.152 \mathrm{M}$ glycine (SERVA Electrophoresis $\mathrm{GmbH}$ ), $0.1 \%$ SDS, diluted from $10 \mathrm{x}$ stock). Gels were stained with Coomassie staining solution $(0.2 \%$ Coomassie Brilliant Blue (SERVA Electrophoresis $\quad \mathrm{GmbH}), \quad 45 \%$ methanol (Interchema AntonidesInterchema), $10 \%$ acetic acid (Biosolve) and 55\% demineralised water) and destained using destaining solution $(30 \%$ methanol, $10 \%$ acetic acid and $60 \%$ demineralised water).

\section{Formation of Tau fibrils}

Monomeric human full-length Tau was aggregated by adapting a published procedure (34). Briefly, $50 \mu \mathrm{M}$ of Tau were mixed with varying concentrations of heparin $(0.125,1.25,12.5$ and $125 \mu \mathrm{M})$ in 25 $\mathrm{mM}$ HEPES-KOH $\mathrm{pH} 7.5,75 \mathrm{mM}$ $\mathrm{KCl}, 75 \mathrm{mM} \mathrm{NaCl}, 1 \mathrm{mM}$ DTT and Heparin Low Molecular Weight (Santa Cruz Biotech). Samples were 
heated at $95^{\circ} \mathrm{C}$ for $10^{\prime}$ and supplemented with Complete Protease Inhibitors (1/2 tablet/50 ml) and $1 \mathrm{mM}$ DTT. Aggregation was performed at $37^{\circ} \mathrm{C}$ for shaking in an incubator at $180 \mathrm{rpm}$. Samples were collected at time points described in Fig. 4A.

Monomeric human N-terminally FLAG-tagged Tau-RD was aggregated in $25 \mathrm{mM}$ HEPES-KOH $\mathrm{pH}$ 7.5, Complete Protease Inhibitors (1/2 tablet/50 ml), $75 \mathrm{mM}$ $\mathrm{KCl}, 75 \mathrm{mM} \mathrm{NaCl}, 10 \mathrm{mM}$ DTT and Heparin Low Molecular Weight (Santa Cruz Biotech), concentrations depending on the experiment and Tau:heparin ratio were kept at 4:1. Aggregation was performed at $37^{\circ} \mathrm{C}$, shaking at 180 rpm, and aliquots were flash frozen at time points indicated in the text. Aliquots were then thawed in a water bath at $37^{\circ} \mathrm{C}$ for downstream applications.

\section{ThioflavinT assay}

$\mathrm{N}$-terminally FLAG-tagged Tau-RD $(100 \mu \mathrm{l})$ of varying concentrations , $60 \mu \mathrm{M}$ ThioflavinT (Sigma) and $5 \mu \mathrm{M}$ heparin Low Molecular Weight were aggregated in aggregation buffer in a transparent, lidded Greiner 96-well plate (Sigma-Aldrich). Fluorescent spectra were recorded with a SpectraMax i3 (Molecular Devices) every 10 minutes for 16 hours. 


\section{BIBLIOGRAPHY}

[1]G.K. Wilcock, M.M. Esiri, Plaques, tangles and dementia. A quantitative study, J. Neurol. Sci. 56 (1982) 343-356.

[2]M. Goedert, D.S. Eisenberg, R.A. Crowther, Propagation of Tau Aggregates and Neurodegeneration, Annu. Rev. Neurosci. 40 (2017) 189-210.

[3]A. Sydow, A. Van der Jeugd, F. Zheng, T. Ahmed, D. Balschun, O. Petrova, D. Drexler, L. Zhou, G. Rune, E. Mandelkow, R. D'Hooge, C. Alzheimer, E.M. Mandelkow, Reversibility of Tau-related cognitive defects in a regulatable FTD mouse model, J. Mol. Neurosci. 45 (2011) 432-437.

[4]Y. Wang, E. Mandelkow, Tau in physiology and pathology, Nat. Rev. Neurosci. 17 (2016) 5-21.

[5]A. Bejanin, D.R. Schonhaut, R. La Joie, J.H. Kramer, S.L. Baker, N. Sosa, N. Ayakta, A. Cantwell, M. Janabi, M. Lauriola, J.P. O'Neil, M.L. GornoTempini, Z.A. Miller, H.J. Rosen, B.L. Miller, W.J. Jagust, G.D. Rabinovici, Tau pathology and neurodegeneration contribute to cognitive impairment in Alzheimer's disease, Brain 140 (2017) 3286-3300.

[6]L. Dehmelt, S. Halpain, The MAP2/Tau family of microtubule-associated proteins, Genome Biol. 6 (2005) 204-2004-6-1-204. Epub 2004 Dec 23.

[7]A. Harada, K. Oguchi, S. Okabe, J. Kuno, S. Terada, T. Ohshima, R. SatoYoshitake, Y. Takei, T. Noda, N. Hirokawa, Altered microtubule organization in small-calibre axons of mice lacking tau protein, Nature 369 (1994) 488-491.

[8]H.N. Dawson, A. Ferreira, M.V. Eyster, N. Ghoshal, L.I. Binder, M.P. Vitek, Inhibition of neuronal maturation in primary hippocampal neurons from tau deficient mice, J. Cell. Sci. 114 (2001) 1179-1187.

[9]B.Y. Monroy, D.L. Sawyer, B.E. Ackermann, M.M. Borden, T.C. Tan, K.M. Ori-McKenney, Competition between microtubule-associated proteins directs motor transport, Nat. Commun. 9 (2018) 1487-018-03909-2.

[10]G. Lee, N. Cowan, M. Kirschner, The primary structure and heterogeneity of tau protein from mouse brain, Science 239 (1988) 285-288.

[11]A.W.P. Fitzpatrick, B. Falcon, S. He, A.G. Murzin, G. Murshudov, H.J. Garringer, R.A. Crowther, B. Ghetti, M. Goedert, S.H.W. Scheres, CryoEM structures of tau filaments from Alzheimer's disease, Nature 547 (2017) 185-190.

[12]B. Falcon, W. Zhang, A.G. Murzin, G. Murshudov, H.J. Garringer, R. Vidal, R.A. Crowther, B. Ghetti, S.H.W. Scheres, M. Goedert, Structures of filaments from Pick's disease reveal a novel tau protein fold, Nature 561 (2018) 137-140.

[13]A. Ittner, S.W. Chua, J. Bertz, A. Volkerling, J. van der Hoven, A. Gladbach, M. Przybyla, M. Bi, A. van Hummel, C.H. Stevens, S. Ippati, L.S. Suh, A. Macmillan, G. Sutherland, J.J. Kril, A.P. Silva, J.P. Mackay, A. Poljak, F. Delerue, Y.D. Ke, L.M. Ittner, Site-specific phosphorylation of tau inhibits amyloid-beta toxicity in Alzheimer's mice, Science 354 (2016) 904-908. 
[14]J. Labbadia, R.I. Morimoto, The biology of proteostasis in aging and disease, Annu. Rev. Biochem. 84 (2015) 435-464.

[15]M.D. Mukrasch, S. Bibow, J. Korukottu, S. Jeganathan, J. Biernat, C. Griesinger, E. Mandelkow, M. Zweckstetter, Structural Polymorphism of 441-Residue Tau at Single Residue Resolution, Plos Biology 7 (2009) 399-414.

[16]G.E. Karagöz, A.M.S. Duarte, E. Akoury, H. Ippel, J. Biernat, T. Morán Luengo, M. Radli, T. Didenko, B.A. Nordhues, D.B. Veprintsev, C.A. Dickey, E. Mandelkow, M. Zweckstetter, R. Boelens, T. Madl, S.G.D. Rüdiger, Hsp90-Tau complex reveals molecular basis for specificity in chaperone action, Cell 156 (2014) 963-974.

[17]D.M. Holtzman, M.C. Carrillo, J.A. Hendrix, L.J. Bain, A.M. Catafau, L.M. Gault, M. Goedert, E. Mandelkow, E.M. Mandelkow, D.S. Miller, S. Ostrowitzki, M. Polydoro, S. Smith, M. Wittmann, M. Hutton, Tau: From research to clinical development, Alzheimers Dement. 12 (2016) 10331039.

[18]V.G. KrishnaKumar, S. Gupta, Simplified method to obtain enhanced expression of tau protein from E. coli and one-step purification by direct boiling, Prep. Biochem. Biotechnol. 47 (2017) 530-538.

[19]S. Barghorn, J. Biernat, E. Mandelkow, Purification of recombinant tau protein and preparation of Alzheimer-paired helical filaments in vitro, Methods Mol. Biol. 299 (2005) 35-51.

[20]L.J. Blair, J.D. Baker, J.J. Sabbagh, C.A. Dickey, The emerging role of peptidyl-prolyl isomerase chaperones in tau oligomerization, amyloid processing, and Alzheimer's disease, J. Neurochem. 133 (2015) 1-13.

[21]C. Sato, N.R. Barthelemy, K.G. Mawuenyega, B.W. Patterson, B.A. Gordon, J. Jockel-Balsarotti, M. Sullivan, M.J. Crisp, T. Kasten, K.M. Kirmess, N.M. Kanaan, K.E. Yarasheski, A. Baker-Nigh, T.L.S. Benzinger, T.M. Miller, C.M. Karch, R.J. Bateman, Tau Kinetics in Neurons and the Human Central Nervous System, Neuron 97 (2018) 1284-1298.e7.

[22]D. Fischer, M.D. Mukrasch, J. Biernat, S. Bibow, M. Blackledge, C. Griesinger, E. Mandelkow, M. Zweckstetter, Conformational Changes Specific for Pseudophosphorylation at Serine 262 Selectively Impair Binding of Tau to Microtubules, Biochemistry (N. Y. ) 48 (2009) 1004710055.

[23]N. Gustke, B. Trinczek, J. Biernat, E.M. Mandelkow, E. Mandelkow, Domains of Tau-Protein and Interactions with Microtubules, Biochemistry (N. Y. ) 33 (1994) 9511-9522.

[24]S.L. Shammas, G.A. Garcia, S. Kumar, M. Kjaergaard, M.H. Horrocks, N. Shivji, E. Mandelkow, T.P. Knowles, E. Mandelkow, D. Klenerman, A mechanistic model of tau amyloid aggregation based on direct observation of oligomers, Nat. Commun. 6 (2015) 7025.

[25]M.M. Mocanu, A. Nissen, K. Eckermann, I. Khlistunova, J. Biernat, D. Drexler, O. Petrova, K. Schonig, H. Bujard, E. Mandelkow, L. Zhou, G. 
Rune, E.M. Mandelkow, The potential for beta-structure in the repeat domain of Tau protein determines aggregation, synaptic decay, neuronal loss, and coassembly with endogenous Tau in inducible mouse models of tauopathy, Journal of Neuroscience 28 (2008) 737-748.

[26]M. Goedert, R. Jakes, M.G. Spillantini, M. Hasegawa, M.J. Smith, R.A. Crowther, Assembly of microtubule-associated protein tau into Alzheimer-like filaments induced by sulphated glycosaminoglycans, Nature 383 (1996) 550-553.

[27]M.v. Bergen, S. Barghorn, J. Biernat, E.M. Mandelkow, E. Mandelkow, Tau aggregation is driven by a transition from random coil to beta sheet structure, Biochimica Et Biophysica Acta-Molecular Basis of Disease 1739 (2005) 158-166.

[28]S.A. Mok, C. Condello, R. Freilich, A. Gillies, T. Arhar, J. Oroz, H. Kadavath, O. Julien, V.A. Assimon, J.N. Rauch, B.M. Dunyak, J. Lee, F.T.F. Tsai, M.R. Wilson, M. Zweckstetter, C.A. Dickey, J.E. Gestwicki, Mapping interactions with the chaperone network reveals factors that protect against tau aggregation, Nat. Struct. Mol. Biol. 25 (2018) 384-393.

[29]G. Ramachandran, J.B. Udgaonkar, Understanding the kinetic roles of the inducer heparin and of rod-like protofibrils during amyloid fibril formation by Tau protein, J. Biol. Chem. 286 (2011) 38948-38959.

[30]K.A. Churion, S.E. Bondos, Identifying solubility-promoting buffers for intrinsically disordered proteins prior to purification, Methods Mol. Biol. 896 (2012) 415-427.

[31]L.P. Kozlowski, Proteome-pl: proteome isoelectric point database, Nucleic Acids Res. 45 (2017) D1112-D1116.

[32]A.M. Livernois, D.J. Hnatchuk, E.E. Findlater, S.P. Graether, Obtaining highly purified intrinsically disordered protein by boiling lysis and single step ion exchange, Anal. Biochem. 392 (2009) 70-76.

[33]L. Ferrari, W.J.C. Geerts, M. van Wezel, R. Kos, A. Konstantoulea, L.S. van Bezouwen, F.G. Förster, S.G.D. Rüdiger, Human chaperones untangle fibrils of the Alzheimer protein Tau, Biorxiv (2018) .

[34]E. Akoury, M. Gajda, M. Pickhardt, J. Biernat, P. Soraya, C. Griesinger, E. Mandelkow, M. Zweckstetter, Inhibition of tau filament formation by conformational modulation, J. Am. Chem. Soc. 135 (2013) 2853-2862. 